Pesq. Vet. Bras. 35(7):677-684, julho 2015 DOI: $10.1590 / \mathrm{S} 0100-736 \mathrm{X} 2015000700013$

\title{
Avaliação dos efeitos de 5-hidroxitriptofano e m-hidroxibenzilhidrazine associados a Lactobacillus spp. na morfometria intestinal e imunomarcação de serotonina em frangos de corte desafiados com Salmonella Enteridis ${ }^{1}$
}

\author{
Taís C. Donato ${ }^{2 *}$, Ana Angelita S. Baptista ${ }^{2}$, Bruna D. Smaniotto ${ }^{2}$, Keila C.O.D. \\ Garcia $^{2}$, Adriano S. Okamoto ${ }^{2}$, Julio L. Sequeira ${ }^{2}$ e Raphael L. Andreatti Filho ${ }^{2}$
}

\begin{abstract}
Donato T.C., Baptista A.A.S., Smaniotto B.D., Garcia K.C.O.D., Okamoto A.S., Sequeira J.L. \& Andreatti Filho R.L. 2015. [Assessment of the effects of 5-hydroxytryptophan and m-hydroxybenzyl hydrazine associated with Lactobacillus spp. in intestinal morphology and immunostaining of serotonin in broilers challenged with Salmonella Enteritidis.] Avaliação dos efeitos de 5-hidroxitriptofano e m-hidroxibenzilhidrazine associados a Lactobacillus spp. na morfometria intestinal e imunomarcação de serotonina em frangos de corte desafiados com Salmonella Enteritidis. Pesquisa Veterinária Brasileira 35(7):677-684. Departamento de Clínica Veterinária, Faculdade de Medicina Veterinária e Zootecnia, Universidade Estadual Paulista, Distrito de Rubião Junior s/n, Botucatu, SP 18608-970, Brazil. E-mail: tais.donato@yahoo.com.br

Enterochromaffin cells are components of the intestinal mucosa to release serotonin lumen, promoting cell growth and secretory activity of various tissues, including intestinal villi. This study evaluated the influence of 5-hydroxytryptophan (5HTP) and m-hidroxibenzilhidrazine (NSD1015) associated with Lactobacillus spp. on body weight and development of intestinal villi in the proximal duodenum of broilers challenged with Salmonella Enteritidis. It was found that the presence of Lactobacillus spp. and Salmonella Enteritidis influenced immunostaining of serotonin in the duodenum. The study was divided into two experiments with and without challenge by $S$. Enteritidis. In Experiment 1, birds without challenge, body weights did not differ significantly ( $p>0.05$ ), and in Experiment 2 , the treatments with precursor and precursor associated with Lactobacillus spp. determined higher body weight of the birds. In both experiments the birds treated with 5HTP showed increased density and villus height in the duodenum, suggesting the presence of 5HTP as a trophic agent. The use of Lactobacillus spp. also determined greater duodenal villus height. The immunostaining of serotonin, birds treated with Lactobacillus spp. in Experiment 1 , and the birds treated with Lactobacillus spp. and challenged with $S$. Enteritidis in Eperiment 2 showed higher values, suggesting that the presence of these bacteria promotes greater release of serotonin into the duodenum. The exact mechanism of how this process occurs needs to be further elucidated.
\end{abstract}

INDEX TERMS: Serotonin, Salmonella Enteritidis, Lactobacillus spp., intestinal morphology, broilers.

RESUMO.- As células enterocromafins são um dos componentes da mucosa intestinal que liberam serotonina para o lúmen, promovendo atividades secretórias e crescimento

\footnotetext{
${ }^{1}$ Recebido em 6 de novembro de 2014.

Aceito para publicação em 8 de julho de 2015.

${ }^{2}$ Departamento de Clínica Veterinária, Faculdade de Medicina Veterinária e Zootecnia (FMVZ), Universidade Estadual Paulista (Unesp), Distrito de Rubião Junior s/n, Botucatu, SP 18608-970, Brasil. *Autor para correspondência: tais.donato@yahoo.com.br
}

celular de vários tecidos, incluindo vilosidades intestinais. 0 presente estudo avaliou as influências do 5-hidroxitriptofano (5HTP) e do m-hidroxibenzilhidrazine (NSD1015), associados a Lactobacillus spp., sobre o peso corporal e o desenvolvimento das vilosidades intestinais na porção proximal do duodeno de frangos de corte desafiados com Salmonella Enteritidis. Verificou-se também se a presença de Lactobacillus spp. e Salmonella Enteritidis influenciaram a imunomarcação de serotonina no duodeno e, para isso, o 
estudo foi dividido em dois experimentos, com e sem desafio por $S$. Enteritidis. No Experimento 1, em aves sem desafio, os pesos corporais não diferiram significantemente ( $p>0,05)$ e, no Experimento 2, aves com desafio, os tratamentos com o precursor isolado e associado a Lactobacillus spp. determinaram maior peso corporal das aves. Nos dois experimentos, as aves tratadas com 5HTP apresentaram aumento na densidade e altura das vilosidades no duodeno, sugerindo a atuação de 5HTP como um agente trófico. A administração de Lactobacillus spp. também determinou altura maior de vilosidades duodenais. Quanto a imunomarcação de serotonina, as aves tratadas com Lactobacillus spp. no Experimento $1 \mathrm{e}$ as aves tratadas com Lactobacillus spp. e desafiadas com $S$. Enteritidis no Experimento 2, apresentaram valores superiores aos demais tratamentos, sugerindo que a presença destas bactérias promove maior liberação de serotonina para o duodeno, porém o mecanismo exato de como este processo ocorre necessita ser mais elucidado.

TERMOS DE INDEXAÇÃO: Serotonina, Salmonella Enteritidis, Lactobacillus spp., morfometria intestinal, frangos de corte.

\section{INTRODUÇÃO}

Aves recém-nascidas possuem um sistema digestório anatomicamente completo, mas funcionalmente incompleto o que leva o trato gastrintestinal a sofrer alterações morfológicas e fisiológicas buscando maior eficiência na digestão e absorção de nutrientes (Murarolli 2008).

As taxas de digestão e absorção intestinal estão diretamente relacionadas com a proliferação e diferenciação celular. À medida que as células indiferenciadas presentes na cripta sofrem mitoses, as células resultantes são deslocadas para a região basal dos vilos, originando as células caliciformes, enterócitos e células enteroendócrinas, que por sua vez são deslocadas para a região apical, onde se desprendem e caem no interior da luz intestinal, promovendo contínua migração de células (Cunningham 2004).

0 equilíbrio entre estes dois processos (perda e proliferação celular) assegura a manutenção do número de células e a capacidade funcional do epitélio. Se ocorrer aumento na taxa de proliferação com ausência, diminuição ou manutenção da perda haverá aumento no número de células e consequentemente, aumento na altura e densidade dos vilos, resultando em maior taxa de digestão e absorção (Maiorka 2001, Furlan et al. 2004).

0 epitélio intestinal atua como uma barreira natural que protege o organismo de bactérias patogênicas e substâncias tóxicas presentes na luz intestinal. Distúrbios na microbiota intestinal causados por patógenos, como por exemplo, Salmonella spp., podem modificar a permeabilidade intestinal e facilitar a invasão destes micro-organismos, alterando o metabolismo, capacidade de digestão e absorção de nutrientes (Deitch et al. 1995, Alverdy et al. 1990, Pelicano et al. 2003).

As bactérias probióticas, como Lactobacillus spp., aumentam a barreira natural do epitélio intestinal contra bactérias patogênicas ou substâncias tóxicas a que o hospedeiro possa ser exposto, promovem a redução de reações inflamatórias e regulam a motilidade intestinal, favorecendo a absorção de nutrientes (Ferreira et al. 1998, Schultz et al. 2004).

A serotonina, uma monoamina biogênica derivada do triptofano, é outro componente importante da mucosa intestinal (Manocha \& Khan 2012). Sua liberação para o lúmen ou lâmina própria faz com que atue sobre células entéricas do sistema nervoso e inicie atividades secretórias e crescimento celular de vários tecidos, incluindo vilosidades e criptas intestinais (Fukumoto et al. 2003, Hasler 2009, Khan \& Ghia 2010).

Diante disso, este estudo avaliou os efeitos do 5-hidroxitriptofano (5HTP) e do m-hidroxibenzilhidrazine (NSD1015), precursor e inibidor da serotonina, respectivamente, associados a Lactobacillus spp. sobre o peso corporal e desenvolvimento das vilosidades intestinais na porção proximal do duodeno de frangos de corte desafiados com Salmonella Enteritidis. Observou-se também a influência de Lactobacillus spp. e $S$. Enteritidis na imunomarcação de serotonina no duodeno.

\section{MATERIAL E MÉTODOS}

\section{Cultivo e administração do inóculo de Lactobacillus spp.}

As cepas de Lactobacillus spp. utilizadas neste estudo (Lactobacillus plantarum, Lactobacillus reuteri, Lactobacillus acidophillus, Lactobacillus brueckii e Lactobacillus spp.) foram isoladas de aves e selecionadas de acordo com a capacidade de adesão e efeito imunomodulatório, descrito por Rocha et al. (2012).

0 cultivo foi realizado separadamente em $15 \mathrm{~mL}$ de meio líquido Man Rogosa Sharp (MRS), em condições de anaerobiose a $37^{\circ} \mathrm{C}$ por 48 horas. Um pool das espécies cultivadas foi formado com $1 \mathrm{~mL}$ de cada cultivo, seguindo a proporção de 1:1. Realizou-se a quantificação de unidades formadoras de colônia (UFC/mL) por meio de diluições decimais seriadas em solução tampão de salina fosfatada (PBS) com pH 7,2 e posterior plaqueamento em ágar MRS.

\section{Cultivo e administração do inóculo de Salmonella Enteritidis}

0 desafio foi feito com cultura de Salmonella enterica subespécie enterica sorotipo Enteritidis fagotipo 4, mutante resistente ao ácido nalidíxico ( $\mathrm{Nal}$ ) e rifampicina (Rif), desenvolvida por meio de cultivos sucessivos em ágar verde brilhante (AVB) contendo ácido nalidíxico $(100 \mu \mathrm{g} / \mathrm{mL}$ de meio) e rifampicina $(100 \mu \mathrm{g} / \mathrm{mL}$ de meio), conforme Andreatti Filho et al. (1997).

$S$. Enteritidis foi cultivada em $20 \mathrm{~mL}$ de caldo infusão de cérebro e coração (BHI) incubada a $40^{\circ} \mathrm{C}$ por 18 horas. A quantificação de unidades formadoras de colônia (UFC) do inóculo foi realizada por meio de diluições decimais seriadas em solução tampão de salina fosfatada (PBS) com pH 7,2 e posterior plaqueamento, em duplicata, em ágar verde brilhante (AVB) contendo ácido nalidíxico $(100 \mu \mathrm{g} / \mathrm{mL}$ de meio) e rifampicina $(100 \mu \mathrm{g} / \mathrm{mL}$ de meio).

\section{5-hidroxitriptofano (5HTP) em-hidroxibenzilhidrazine (NSD1015)}

Estudos prévios foram realizados no Infectório do Laboratório de Ornitopatologia da Faculdade de Medicina Veterinária e Zootecnia da UNESP/Botucatu-SP, com o objetivo de determinar as doses de 5-hidroxitriptofano (5HTP) e m-hidroxibenzilhidrazine (NSD1015). Optou-se por administrar 5HTP na dose de 25mg/ kgPV e NSD1015 na dose de 150mg/kgPV.

Os fármacos foram administrados via intraperitoneal na quantidade de $0,1 \mathrm{ml}$ /ave com seringa de $1 \mathrm{~mL}$ e agulha $0,45 \times 13 \mathrm{~mm}$. As inoculações foram realizadas em ângulo de $45^{\circ}$ no quadrante 
médio da parede pélvica, conforme descrito por Cedraz-Mercez et al. (2007).

\section{Delineamento experimental}

Experimento 1. Duzentos e oitenta e oito machos matrizes de corte com um dia de vida foram distribuídos em um delineamento experimental inteiramente casualizado, composto por seis tratamentos de 36 aves cada, oito períodos de avaliação e seis repetições por período:

Tratamento 1 (T1): aves tratadas com 5HTP;

Tratamento 2 (T2): aves tratadas com NSD1015;

Tratamento 3 (T3): aves tratadas com 5HTP e pool de Lactobacillus;

Tratamento 4 (T4): aves tratadas com NSD1015 e pool de Lactobacillus;

Tratamento 5 (T5): aves tratadas com pool de Lactobacillus;

Tratamento 6 (T6): aves tratadas com $0,1 \mathrm{~mL}$ de $\mathrm{NaCl}$ a $0,9 \%$.

Administrou-se $25 \mathrm{mg} / \mathrm{kg}$ de peso vivo de 5-hidroxitriptofano, $150 \mathrm{mg} / \mathrm{kg}$ de peso vivo de $\mathrm{m}$-hidroxibenzilhidrazine e $\mathrm{NaCl}$ uma vez ao dia, via intraperitoneal, por cinco dias consecutivos (do $4^{\circ}$ o ao $8^{\circ}$ dia de idade), na quantidade de $0,1 \mathrm{~mL} /$ ave com seringa de $1 \mathrm{~mL}$ e agulha de $0,45 \times 13 \mathrm{~mm}$.

0 pool de Lactobacillus contendo $1,0 \times 10^{9} \mathrm{UFC} / \mathrm{mL}$ foi administrado uma vez ao dia, via oral, diretamente no esôfago/inglúvio de cada ave, com o auxílio de agulha de gavagem estéril, por cinco dias consecutivos (do $4^{\circ}$ ao $8^{\circ}$ dia de idade), na quantidade de $500 \mu \mathrm{L} /$ ave.

Experimento 2. Trezentos e sessenta e seis machos matrizes de corte com um dia de vida foram distribuídos em um delineamento experimental inteiramente casualizado, composto por sete tratamentos de 36 aves cada, oito períodos de avaliação e seis repetições por período:

Tratamento 1 (T1): aves tratadas com 5HTP e desafiadas com $S$. Enteritidis;

Tratamento 2 (T2): aves tratadas com NSD1015 e desafiadas com $S$. Enteritidis;

Tratamento 3 (T3): aves tratadas com 5HTP, pool de Lactobacillus e desafiadas com $S$. Enteritidis;

Tratamento 4 (T4): aves tratadas com NSD1015, pool de Lactobacillus e desafiadas com $S$. Enteritidis;

Tratamento 5 (T5): aves tratadas com pool de Lactobacillus e desafiadas com $S$. Enteritidis;

Tratamento 6 (T6): aves desafiadas com $S$. Enteritidis;

Tratamento 7 (T7): aves tratadas com $0,1 \mathrm{~mL}$ de $\mathrm{NaCl}$ a $0,9 \%$.

5-hidroxitriptofano (5HTP), m-hidroxibenzilhidrazine (NSD 1015) e $\mathrm{NaCl}$ foram administradas uma vez ao dia, via intrape-

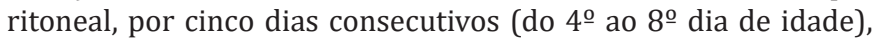
na quantidade de $0,1 \mathrm{~mL} /$ ave com seringa de $1 \mathrm{~mL}$ e agulha de $0,45 \times 13 \mathrm{~mm}$.

0 pool de Lactobacillus contendo $1,0 \times 10^{9} \mathrm{UFC} / \mathrm{mL}$ foi administrado uma vez ao dia, via oral, por cinco dias consecutivos (do $4^{\circ}$ ao $8^{\circ}$ dia de idade), na quantidade de $500 \mu \mathrm{l} /$ ave com o auxílio de agulha de gavagem estéril.

0 desafio com $S$. Enteritidis $\left(2,0 \times 10^{6} \mathrm{UFC} / \mathrm{mL}\right)$ foi realizado no oitavo dia de idade, via oral na quantidade de $1 \mathrm{~mL} /$ ave com auxílio de agulha de gavagem estéril.

\section{Avaliação do peso médio corporal}

Para esta avaliação, seis aves de cada tratamento foram pesadas com o uso de balança digital nos dias quatro, oito, 10, 12, 14, 21,28 e 35 de idade das aves.
Coleta de duodeno para avaliação das vilosidades intestinais e ensaios de imunoistoquímica

Quatro aves de cada tratamento foram eutanasiadas por deslocamento cervical (conforme aprovação do comitê de ética em experimentação animal da FMVZ/Unesp, protocolo no 191/2011CEUA) e amostras individuais de, aproximadamente, um centímetro de comprimento da porção proximal do duodeno foram coletadas, abertas pela borda mesentérica, estendidas pela túnica serosa, lavadas com solução tampão de salina fosfatada (PBS) com pH 7,2 (para retirar o excesso de fezes), e fixadas em formalina a $10 \%$ por 24 horas. Posteriormente, o material foi lavado em água destilada e acondicionado em álcool etílico $70 \%$ até a preparação histológica.

\section{Mensuração da altura e densidade das vilosidades}

Após acondicionamento das amostras em álcool etílico 70\%, estas foram desidratadas em série crescente de álcool etílico, recortadas, diafanizadas em xilol e incluídas em parafina. Em cada lâmina histológica foram colocados seis cortes semi-seriados com $5 \mu \mathrm{m}$ de espessura, corados segundo a técnica hematoxilina-eosina-HE (Behmer et al. 2003). 0 estudo morfométrico da altura das vilosidades duodenais $(\mu \mathrm{m})$ foi realizado utilizando sistema analisador de imagem Axio Vision Software Rel. Versão 4.6.1 (Zeiss Vision) acoplado a microsópio óptico modelo Axio Imager A1, Zeiss.

Foram efetuadas trinta medidas da altura das vilosidades para cada segmento de duodeno coletado, totalizando a leitura de 120 vilos por tratamento. As medidas de altura das vilosidades foram tomadas a partir da região basal, que coincide com a porção superior das criptas até o ápice.

Para a mensuração do número de vilos, as amostras foram fotografadas em cinco campos distintos, com objetiva $5 \mathrm{X}$, totalizando 20 fotos por tratamento. Os vilos foram contados de acordo com o campo de captura (Schwarz et al. 2011).

\section{Imunoistoquímica}

A técnica de imunoistoquímica baseou-se no protocolo utilizado por Masseno (2012) e estabelecido pelo Laboratório de Pesquisa do Serviço de Patologia Veterinária da Faculdade de Medicina Veterinária e Zootecnia da Unesp-Botucatu/SP.

As lâminas foram confeccionadas com segmentos de duodeno de $3 \mu \mathrm{m}$ de espessura e incubadas a $56^{\circ} \mathrm{C}$ por 24 horas permitindo adesão adequada do tecido a lâmina.

Os cortes histológicos foram desparafinizados com xilol, re-hidratados em álcool etílico em concentrações decrescentes e água deionizada. A recuperação antigênica foi realizada com tampão Citrato (10mM, pH 6,0), em panela de pressão (Pascal ${ }^{\circledR} /$ Dako) durante 30 segundos a $125^{\circ} \mathrm{C}$. Em seguida, as lâminas foram resfriadas, lavadas em água deionizada, incubadas por 30 minutos em peróxido de hidrogênio 3\% (diluído em Metanol), lavadas em água deionizada e incubadas em tampão TRIS pH 7,4 por 10 minutos. Os cortes foram submetidos ao bloqueio de proteínas inespecíficas com soro albumina bovina a 5\% durante 1 hora a $27^{\circ}$ C. 0 excesso de solução foi retirado e incubou-se cada lâmina com o anticorpo primário anti-serotonin antibody (S5545; Sigma-Aldrich, USA) na diluição de 1:1200 durante 18 horas (overnight) em câmara úmida a $4^{\circ} \mathrm{C}$.

Posteriormente, as lâminas foram lavadas com tampão TRIS pH 7,4 por 5 minutos. Aplicou-se $100 \mu \mathrm{L}$ do reagente Post Primary Block do sistema de visualização Novolink ${ }^{\mathrm{TM}}$ Max Polymer Detection. As lâminas foram incubadas a $27^{\circ} \mathrm{C}$ por 30 minutos, lavadas com TRIS pH 7,4 e 100 $\mu \mathrm{L}$ do reagente Max Polymer foi aplicado, sendo as mesmas incubadas novamente por 30 minutos a $27^{\circ} \mathrm{C}$. Em seguida foram lavadas com TRIS pH 7,4. 
Para vizualização da reação, as lâminas foram tratadas com DAB Chromogen, do sistema de visualização Novolink ${ }^{\mathrm{TM}}$ Max Polymer Detection, RE7150-CE, por 3 minutos. Os cortes foram contra-corados com Hematoxilina de Harris e desidratados em concentrações crescentes de álcool e xilol.

Para a mensuração da imunomarcação de serotonina, as lâminas foram fotografadas em vinte campos distintos, com objetiva 40X, totalizando 80 fotos por tratamento. A visualização foi feita utilizando sistema analisador de imagem Axio Vision Software Rel. Versão 4.6.1 (Zeiss Vision) acoplado a microsópio óptico modelo Axio Imager A1, Zeiss.

\section{Análise estatística}

O delineamento experimental foi inteiramente casualizado, utilizando-se esquema fatorial de grupos independentes. Os resultados foram submetidos à análise de variância seguida de teste de Tukey a $5 \%$ de significância pelo procedimento PROC MIXED (SAS Institute 2009).

\section{RESULTADOS}

O peso médio corporal das aves do Experimento 1 está expresso na Figura 1. Os tratamentos apresentaram concentrações similares, sem diferenças significativas $(p>0,05)$ até 28 dias de idade. A média inicial do peso das aves foi de aproximadamente $55,67 \mathrm{~g}$ e apresentou aumento significativo $(\mathrm{p}<0,05)$ até 35 dias, com valores de 1906,33g.

Aos 35 dias, o tratamento T1 determinou o maior peso corporal $(2105,17 \mathrm{~g})$ e diferiu significativamente dos demais tratamentos. Os tratamentos T2 e T4 apresentaram peso corporal de 1890,33g e 1939,83g, respectivamente, com diferença significativa em relação ao controle negativo (T6), com peso de $1748,17 \mathrm{~g}$.

No Experimento 2, os tratamentos apresentaram concentrações similares de peso médio corporal sem diferenças significativas ( $p>0,05)$ até os 14 dias de idade (Fig.2). Aos 21 dias, o T1 com maior peso corporal $(817,83 \mathrm{~g})$ apresentou diferença estatística significativa em relação ao T5, menor peso $(682,50 \mathrm{~g})$. Aos 28 e 35 dias os tratamentos T1 e T3 diferiram do controle negativo (T7).

A densidade e altura das vilosidades da porção proximal do duodeno, no Experimento 1, estão expressas no Quadro 1.
Experimento 1 - Peso corporal das aves

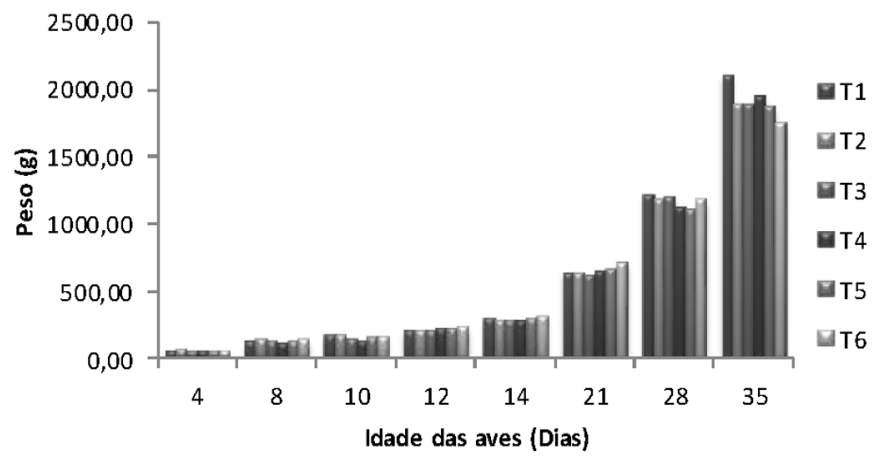

Fig.1. Peso médio corporal de frangos de corte após administração de 5-hidroxitriptofano (5HTP) e do m-hidroxibenzilhidrazine (NSD1015) sem desafio com Salmonella Enteritidis (Experimento 1). Tratamentos: T1 (5-hidroxitriptofano); T2 (NSD1015); T3 (5-hidroxitriptofano + pool de Lacotbacillus); T4 (NSD1015 + pool de Lactobacillus); T5 (pool de Lactobacillus); T6 (controle negativo).

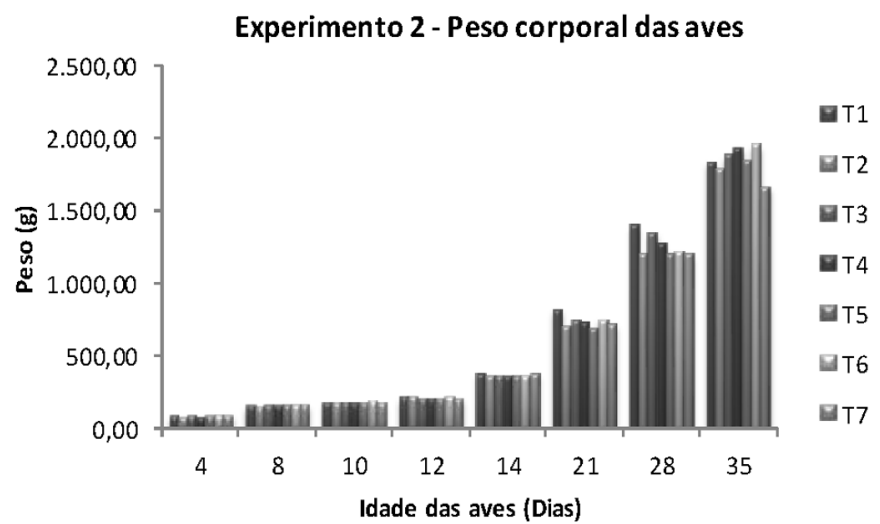

Fig.2. Peso médio corporal de frangos de corte após administração de 5-hidroxitriptofano (5HTP) e do m-hidroxibenzilhidrazine (NSD1015) com desafio por Salmonella Enteritidis (Experimento 2). Tratamentos: T1 (5-hidroxitriptofano $+S$. Enteritidis); T2 (NSD1015+ S. Enteritidis); T3 (5-hidroxitriptofano + pool de Lacotbacillus + S. Enteritidis); T4 (NSD1015 + pool de Lactobacillus + S. Enteritidis); T5 (pool de Lactobacillus + S. Enteritidis); T6 (S. Enteritidis); T7 (controle negativo).

Quadro 1. Valores médios de densidade (número vilos/campo) e altura dos vilos $(\mu \mathrm{m})$ no duodeno de frangos de corte, após administração de 5-hidroxitriptofano (5HTP) e do m-hidroxibenzilhidrazine (NSD1015) sem desafio com Salmonella Enteritidis (Experimento 1). Tratamentos: T1 (5-hidroxitriptofano); T2 (NSD1015); T3 (5-hidroxitriptofano + pool de Lacotbacillus); T4 (NSD1015 + pool de Lactobacillus); T5 (pool de Lactobacillus); T6 (controle negativo)

\begin{tabular}{|c|c|c|c|c|c|c|c|c|}
\hline \multirow[t]{3}{*}{ Tratamentos } & \multicolumn{6}{|c|}{ Idade (Dias) } & \multicolumn{2}{|c|}{ Médias* } \\
\hline & \multicolumn{2}{|c|}{14 dias } & \multicolumn{2}{|c|}{21 dias } & \multicolumn{2}{|c|}{28 dias } & Densidad & e Altura \\
\hline & Densidade & Altura & Densidade & Altura & Densidade & Altura & & \\
\hline $\mathrm{T} 1$ & $11,2^{\mathrm{Bc} *}$ & $1253,8^{\mathrm{Ab} *}$ & $7,1^{\text {Ad }}$ & $1608,6^{\mathrm{Bb}}$ & $5,45^{\mathrm{Ab}}$ & $1716,1^{\mathrm{Bb}}$ & $9,7^{\mathrm{e}}$ & $1526,1^{c}$ \\
\hline $\mathrm{T} 2$ & $10,5^{\mathrm{Cc}}$ & $1096,3^{\mathrm{Aab}}$ & $7,1^{\mathrm{Bab}}$ & $1405,3^{\mathrm{Bab}}$ & $5,45^{\text {Аa }}$ & $1535,9^{\mathrm{Bab}}$ & $7,7^{\mathrm{bc}}$ & $1345,8^{\mathrm{ab}}$ \\
\hline T3 & $8,9^{\mathrm{Bb}}$ & $1300,3^{\mathrm{Ab}}$ & $8,4^{\mathrm{ABcd}}$ & $1471,7^{\text {Bab }}$ & $7,60^{\mathrm{Ab}}$ & $1586,0^{\mathrm{Bab}}$ & $8,3^{\mathrm{cd}}$ & $1452,7^{\mathrm{bc}}$ \\
\hline $\mathrm{T} 4$ & $8,5^{\mathrm{cb}}$ & $1123,7^{\mathrm{Aab}}$ & $7,3^{\text {Babc }}$ & $1405,3^{\text {Bab }}$ & $5,95^{\mathrm{Aa}}$ & $1482,2^{\mathrm{Ba}}$ & $7,3^{b}$ & $1337,0^{\mathrm{ab}}$ \\
\hline T5 & $10,4^{\mathrm{Bc}}$ & $1231,9^{\text {Aab }}$ & $7,7^{\mathrm{Abc}}$ & $1423,0^{\mathrm{Bab}}$ & $7,45^{\mathrm{Ab}}$ & $1551,5^{\text {Bab }}$ & $8,5^{d}$ & $1402,1^{\mathrm{ab}}$ \\
\hline T6 & $7,0^{\mathrm{Ba}}$ & $1044,8^{\mathrm{Aa}}$ & $6,5^{\mathrm{Aa}}$ & $1391,2^{\mathrm{Ba}}$ & $5,40^{\mathrm{Aa}}$ & $1477,9^{\mathrm{Ba}}$ & $6,3^{\mathrm{a}}$ & $1304,6^{a}$ \\
\hline Médias* & $9,45^{\mathrm{C}}$ & $1175,13^{\mathrm{A}}$ & $7,75^{\mathrm{B}}$ & $1450,87^{\mathrm{B}}$ & $6,73^{A}$ & $1558,27^{\mathrm{C}}$ & & \\
\hline
\end{tabular}

* Médias seguidas pela mesma letra maiúscula na linha ou minúscula na coluna não diferem estatisticamente pelo Teste de Tukey $(\mathrm{p}<0,05)$. 
Quadro 2. Valores médios de densidade (número vilos/campo) e altura dos vilos $(\mu \mathrm{m})$ no duodeno de frangos de corte, após administração de 5-hidroxitriptofano

(5HTP) e do m-hidroxibenzilhidrazine (NSD1015) com desafio por Salmonella Enteritidis (Experimento 2). Tratamentos: T1 (5-hidroxitriptofano +

$S$. Enteritidis); T2 (NSD1015+ S. Enteritidis); T3 (5-hidroxitriptofano + pool de Lacotbacillus + S. Enteritidis); T4 (NSD1015 + pool de Lactobacillus+ $S$. Enteritidis); T5 (pool de Lactobacillus + S. Enteritidis); T6 (S. Enteritidis); T7 (controle negativo)

\begin{tabular}{|c|c|c|c|c|c|c|c|c|}
\hline \multirow[t]{3}{*}{ Tratamentos } & \multicolumn{6}{|c|}{ Momento - Dias de vida } & \multirow{2}{*}{\multicolumn{2}{|c|}{$\frac{\text { Médias* }}{\text { Densidade Altura }}$}} \\
\hline & \multicolumn{2}{|c|}{14 dias } & \multicolumn{2}{|c|}{21 dias } & \multicolumn{2}{|c|}{28 dias } & & \\
\hline & Densidade & Altura & Densidade & Altura & Densidade & Altura & & \\
\hline $\mathrm{T} 1$ & $9,7^{\mathrm{Bd} *}$ & $1407,0^{\mathrm{Ab} *}$ & $7,8^{A c}$ & $1606,3^{\mathrm{Bd}}$ & $6,9^{\mathrm{Ab}}$ & $1702,1^{\mathrm{Bd}}$ & $8,1^{\mathrm{d}}$ & $1571,8^{\mathrm{b}}$ \\
\hline T2 & $7,1^{\text {Bab }}$ & $1273,6^{\text {Aab }}$ & $6,0^{\mathrm{Aa}}$ & $1359,5 A^{\text {Babc }}$ & $5,2^{\mathrm{Aa}}$ & $1459,9^{\text {Bab }}$ & $6,1^{\mathrm{ab}}$ & $1364,3^{\mathrm{a}}$ \\
\hline T3 & $8,8^{\mathrm{Bcd}}$ & $1381,2^{\mathrm{Ab}}$ & $8,3^{\mathrm{ABC}}$ & $1520,9^{\text {Acd }}$ & $7,2^{\mathrm{Ab}}$ & $1679,4^{\mathrm{Bcd}}$ & $8,1^{\mathrm{d}}$ & $1527,2^{b}$ \\
\hline $\mathrm{T} 4$ & $7,5^{\text {Babc }}$ & $1177,5^{\text {Аа }}$ & $6,2^{\text {Aab }}$ & $1386,4^{\mathrm{Babc}}$ & $5,5^{\mathrm{Aa}}$ & $1483,6^{\text {Babc }}$ & $6,4^{\mathrm{b}}$ & $1349,1^{\mathrm{a}}$ \\
\hline T5 & $8,2^{\mathrm{Bbc}}$ & $1380,2^{\mathrm{Ab}}$ & $7,4^{\mathrm{Bbc}}$ & $1455,7^{\mathrm{Abcd}}$ & $6,1^{\text {Aab }}$ & $1623,9^{\mathrm{Bbcd}}$ & $7,2^{c}$ & $1486,6^{b}$ \\
\hline T6 & $6,3^{\mathrm{Ba}}$ & $1216,7^{\text {Aab }}$ & $5,0^{\mathrm{Aa}}$ & $1245,1^{\mathrm{Aa}}$ & $4,9^{\mathrm{Aa}}$ & $1318,9^{\mathrm{Aa}}$ & $5,4^{\mathrm{a}}$ & $1260,2^{\mathrm{a}}$ \\
\hline T7 & $6,4^{\mathrm{Ba}}$ & $1227,3^{\text {Aab }}$ & $5,8 \mathrm{~A}^{\mathrm{Ba}}$ & $1310,5^{\text {ABab }}$ & $5,0^{A a}$ & $1465,2^{\text {Bab }}$ & $5,7^{\mathrm{ab}}$ & $1334,3^{\mathrm{a}}$ \\
\hline Médias* & $7,72^{\mathrm{C}}$ & $1294,79^{\mathrm{A}}$ & $6,66^{\mathrm{B}}$ & $1412,08^{\mathrm{B}}$ & $5,84^{\mathrm{A}}$ & $1533,29^{\mathrm{C}}$ & & \\
\hline
\end{tabular}

* Médias seguidas pela mesma letra maiúscula na linha ou minúscula na coluna não diferem estatisticamente pelo Teste de Tukey $(\mathrm{p}<0,05)$.

Aos 14 dias, os tratamentos T1, T2 e T5 determinaram as maiores densidades, com valores de 11,2, 10,5 e 10,4, respectivamente, diferindo significativamente dos demais tratamentos. Com 21 e 28 dias, os valores médios da densidade dos vilos nos tratamentos T2 e T4 foram estatisticamente iguais ao controle negativo (T6). 0 tratamento T1 apresentou maior densidade com 14, 21 e 28 dias. Ao avaliar a média geral dos tratamentos, observa-se que os melhores resultados foram determinados pelos tratamentos T1, T3 e T5.

Com relação a altura dos vilos aos 14 dias de idade, T1 e T3 diferiram significativamente do controle negativo (T6). Aos 21 dias, T1 $(1608,6 \mu \mathrm{m})$ diferiu de T6 (1391,2 $\mu \mathrm{m})$. E aos 28 dias, T1 apresentou diferença significativa de T6 e T4. Observa-se nas médias gerais dos tratamentos, que T1 $(1526,15 \mu \mathrm{m})$ e T3 $(1452,7 \mu \mathrm{m})$ apresentaram os melhores resultados e diferiram significativamente de T6 $(1304,6 \mu \mathrm{m})$.

No Experimento 2, os valores das densidade e altura estão expressos no Quadro 2.

Aos 14 dias o T1 apresentou o maior número de vilosidades, sendo estatisticamente igual a T3 e diferente dos demais tratamentos. Aos 21 e 28 dias os T1 e T3 determinaram as maiores densidades e diferiram significativamente dos controles positivo (T6) e negativo (T7). Ao avaliar as médias gerais dos tratamentos, observa-se que os tratamentos T1 e T3 apresentaram as maiores densidades de vilosidades em duodeno, não diferindo entre si, mas diferindo significativamente dos demais tratamentos.

Aos 14 dias de vida, o tratamento T4 apresentou a menor altura de vilosidade duodenal $(1177,5 \mu \mathrm{m})$, diferindo estatisticamente dos tratamentos T1 $(1407,0 \mu \mathrm{m}), \mathrm{T} 3$ $(1381,2 \mu \mathrm{m})$ e T5 $(1380,2 \mu \mathrm{m})$, que apresentaram os maiores valores.

Com 21 e 28 dias, T1 obteve a maior altura, sendo estatisticamente semelhante a T3 e T5, mas com diferença significativa em relação aos demais tratamentos. Ao avaliar a média geral, os tratamentos T1, T3 e T5 não apresentaram diferença significativa e obtiveram valores de altura das vilosidades duodenais mais altos.

As células enterocromafins que imunomarcaram serotonina foram observadas no duodeno de frangos de corte conforme Fig. 3.

Aos 14 e 21 dias o T3 apresentou a maior quantidade de células imunomarcadas, 10,21 e 8,63, respectivamente, diferindo significativamente de todos os tratamentos. Os tratamentos T4 (14d-7,07; 21d-6,63) e T5 (14d-7,56; 21d$6,37)$ apresentaram diferenças significativas em relação ao controle negativo T6 (14d-4,88; 21d-4,68). Com 28 dias de idade, os tratamentos T1 $(5,51), \mathrm{T} 3(6,06), \mathrm{T} 4(6,01)$ e T5 $(6,00)$ foram semelhantes entre si e diferentes do controle negativo T6 $(4,02)$. Ao comparar as médias dos tratamentos, observa-se que todos apresentaram diferenças significativas em relação ao controle negativo (T6), sendo que T3 apresentou o melhor resultado $(8,30)$.

No Experimento 2, os tratamentos T3, T4, T5 e T6 apresentaram maior número de células com imunomarca-

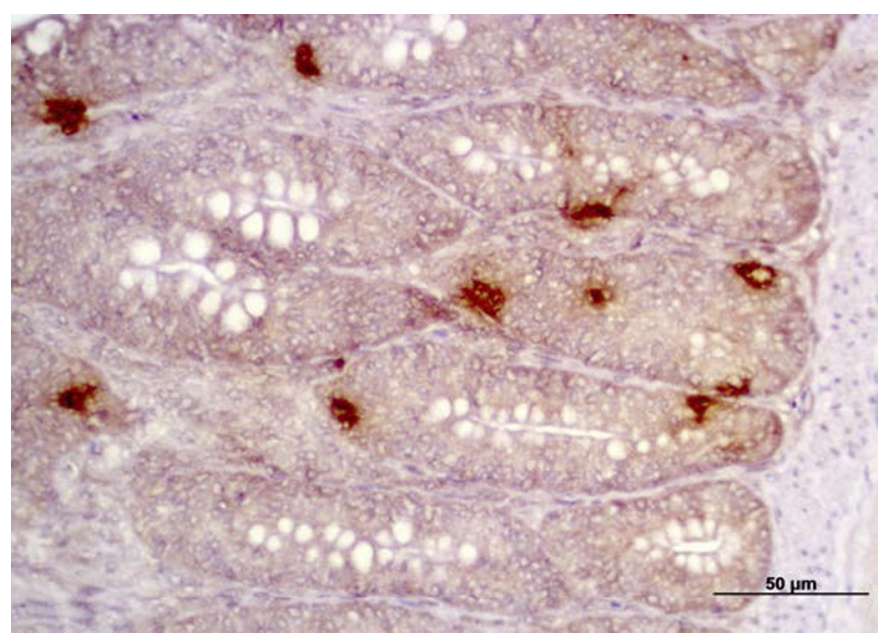

Fig.3. Imunomarcação de serotonina em células enterocromafins de duodeno de frangos de corte aos 14 dias de vida. Barra $=50 \mu \mathrm{m}$. 
Experimento 2 - Imunoistoquímica

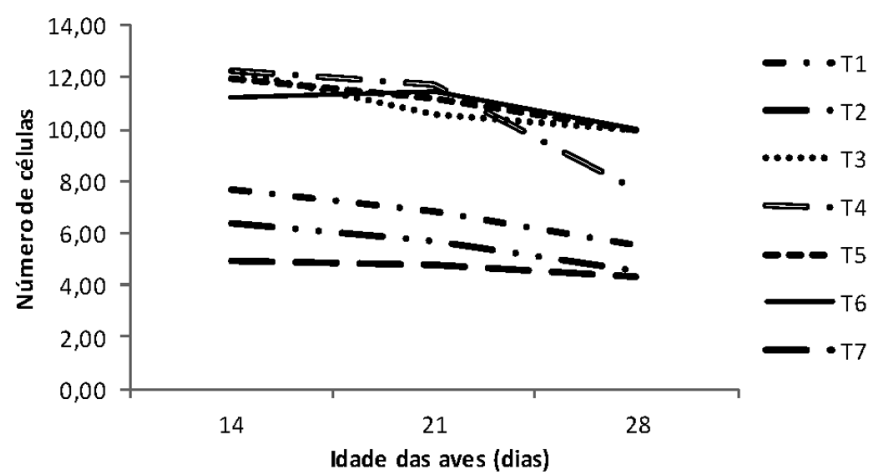

Fig.4. Número de células imunomarcadas para serotonina (número células/campo) em duodeno de frangos de corte, após administração de 5-hidroxitriptofano (5HTP) e do m-hidroxibenzilhidrazine (NSD1015) com desafio por Salmonella Enteritidis (Experimento 2). Tratamentos: T1 (5-hidroxitriptofano $+S$. Enteritidis); T2 (NSD1015+S. Enteritidis); T3 (5-hidroxitriptofano + pool de Lacotbacillus + S. Enteritidis); T4 (NSD1015 + pool de Lactobacillus + S. Enteritidis); T5 (pool de Lactobacillus $+S$. Enteritidis); T6 (S. Enteritidis); T7 (controle negativo).

ção positiva para serotonina, sendo estatisticamente semelhantes $(p>0,05)$ e com diferença significativa em relação aos demais tratamentos $(\mathrm{p}<0,05)$ aos 14 e 21 dias. Com 28 dias os tratamentos T3, T4, T5 e T6 também determinaram contagens de células imunomarcadas maiores, sendo estatisticamente semelhantes entre si, mas diferentes do controle negativo (T7). Com relação às médias gerais dos tratamentos, com exceção do T2 todos os demais tratamentos apresentaram diferenças significativas em relação ao controle negativo (T7) conforme Figura 4.

\section{DISCUSSÃO}

0 crescimento e a manutenção do trato gastrintestinal das aves são fatores que proporcionam melhora na eficiência dos processos digestivos, uma vez que o epitélio intestinal atua como barreira natural protegendo o organismo de bactérias patogênicas e substâncias tóxicas presentes na luz intestinal (Pelicano et al. 2003, Marchini et al. 2009).

0 presente estudo avaliou os efeitos do 5-hidroxitriptofano (5HTP) e m-hidroxibenzilhidrazine (NSD1015) associados à Lactobacillus spp. sobre o peso corporal das aves e o desenvolvimento das vilosidades intestinais na porção proximal do duodeno de frangos de corte desafiados com Salmonella Enteritidis, além de analisar a influência de Lactobacillus spp. e $S$. Enteritidis na imunomarcação de serotonina no duodeno.

Ao analisar os resultados obtidos, nota-se que no Experimento 1 não houve diferença significativa $(p>0,05)$ entre os tratamentos com relação ao peso médio corporal. Já no experimento 2, os tratamentos com o precursor (T1) e o precursor associado a Lactobacillus spp. (T3) determinaram maior peso médio corporal das aves, diferindo significativamente do controle negativo (T7).

Tanto os resultados do Experimento 1 como do Experimento 2 corroboram com estudos prévios realizados em no nosso laboratório, onde a serotonina, seu precursor e inibidor foram administrados em frangos de corte e apesar de algumas diferenças estatísticas não foram observadas grandes alterações no ganho de peso das aves tratadas com o precursor e inibidor.

As aves que receberam o precursor e a associação do precursor com Lactobacillus tiveram leve aumento no peso médio corporal, o que se justifica pelos resultados observados na altura e densidade das vilosidades no duodeno. Segundo Maiorka (2001) e Furlan et al. (2004) se ocorrer um aumento na taxa de proliferação com ausência, diminuição ou manutenção da perda haverá um aumento no número de células, e, consequentemente, um aumento na altura e densidade dos vilos, resultando em uma maior taxa de digestão e absorção, portanto aumento do peso corporal.

Ao comparar a média geral, as aves que receberam somente o pool de Lactobacillus (T5) não diferiram significativamente do controle negativo (T7), corroborando com os dados obtidos por Alvarez et al. (1994) e Corneli (2004), que ao adicionarem probióticos na ração de frangos de corte não encontraram melhora significativa no ganho de peso das aves.

Nos dois experimentos, com e sem desafio por $S$. Enteritidis, as aves tratadas com 5HTP apresentaram aumento na densidade e altura das vilosidades no duodeno.

0 tratamento T1 (5HTP) apresentou o maior número de vilos e altura por campo, seguido pelos tratamentos T3 (5HTP + pool de Lactobacillus) e T5 (pool de Lactobacillus). Estes achados corroboram com os resultados obtidos por Nakamura et al. (2008) que ao administrarem sistemicamente 5HTP e NSD1015, observaram que 5HTP proporcionou aumento na densidade das vilosidades intestinais em testes in vivo realizados em camundongos e in vitro com cultura de linhagens celulares (Caco-2).

No Experimento 2, os tratamentos T1 (5HTP), T3 (5HTP + pool de Lactobacillus) e T5 (pool de Lactobacillus) apresentaram novamente as maiores densidades e alturas de vilos por campo, diferindo significativamente do inibidor (T2), inibidor associado à Lactobacillus (T4), controle positivo (T6) e controle negativo (T7).

Estes resultados sugerem a atuação de 5-hidroxitriptofano como um agente trófico, que segundo Macari \& Maiorka (2000) é definido como uma substância que atua no desenvolvimento intestinal, estimulando o processo mitótico na região cripta:vilo e levando a um aumento na quantidade de células e altura dos vilos.

A administração de Lactobacillus spp. determinou alturas maiores de vilosidades duodenais, corroborando com os resultados obtidos por Pelicano et al. (2003), que ao adicionarem pool de Lactobacillus spp. na água de bebida, observaram maior altura de vilos em duodeno, e por Dobrogosz et al. (1991), que administraram Lactobacillus reuteri na ração de frangos de corte aos 42 dias de vida, observando também vilos maiores. Em nosso estudo o aumento das vilosidades ocasionado pela utilização de Lactobacillus promoveu um aumento no ganho de peso, porém não o suficiente para diferir estatisticamente do controle negativo.

De acordo com Ramos et al. (2011), a maior densidade e altura das vilosidades está relacionada aos resultados de desempenho, em que as aves apresentam maior peso cor- 
poral, devido ao aumento da capacidade de digestão e absorção de nutrientes.

Nas três avaliações, dos dois experimentos, o tratamento T1 (5HTP) apresentou os melhores resultados quanto a densidade de vilos por campo avaliado, altura de vilosidades em duodeno e peso médio corporal das aves, demonstrando a atuação do precursor nos processos de digestão e absorção de nutrientes, bem como confirmando a relação da densidade, altura das vilosidades e desempenho de frangos de corte citados anteriormente.

Quanto a imunomarcação de serotonina, as aves tratadas com Lactobacillus spp. no experimento $1 \mathrm{e}$ as aves tratadas com Lactobacillus spp. e desafiadas com $S$. Enteritidis no Experimento 2, apresentaram valores superiores aos demais tratamentos. Provavelmente a presença de Lactobacillus spp. e sua ação na mucosa intestinal promoveu maior liberação de serotonina. Linden et al. (2003) e 0'Hara et al. (2004) observaram aumento de células imunomarcadas para serotonina em suínos com colite e ilite experimental induzidas pelo ácido trinitrobenzeno sulfônico (TNBS), sugerindo que processos inflamatórios aumentem a liberação de serotonina pelas células enterocromafins.

Porém os mecanismos desta interação entre serotonina e Lactobacillus spp. precisam ser elucidados.

As alterações na imunomarcação positiva de serotonina nem sempre são observadas durante os processos inflamatórios. Acredita-se que estas dependam da espécie animal avaliada e do tipo e grau de inflamação (0`Hara et al. 2006). Sabe-se que determinados processos inflamatórios ocasionam aumento no número de células imunomarcadas para serotonina, mas as causas e os mecanismos deste aumento ainda são desconhecidos.

De acordo com Linden et al. (2003) é possível que o aumento de células imunomarcadas para serotonina ocorra devido a diminuição da sua recaptação pelo seu transportador específico (SERT). Os resultados observados por 0 'Hara et al. (2004) indicam que a recaptação de serotonina é realmente reduzida durante a inflamação por Citrobacter rodentium, onde as células imunomarcadas para SERT foram reduzidas 10 dias após a infecção. Mesmos resultados foram observados em colites ulcerativas em humanos (Coates et al. 2004).

Neste estudo, observou-se aumento na imunomarcação de serotonina no duodeno devido à presença de Lactobacillus spp. e $S$. Enteritidis, que estimularam a mucosa intestinal promovendo maior liberação de serotonina das células enterocromafins, porém mais estudos são necessário para descobrir e elucidar o mecanismo exato de como este processo ocorre.

\section{CONCLUSÃO}

Conclui-se que a administração de 5-hidroxitriptofano isolado ou associado a Lactobacillus spp. determinou maiores densidade e altura das vilosidades no duodeno, bem como Lactobacillus spp. e Salmonella Enteritidis aumentaram a imunoexpressão de serotonina no duodeno. Estes resultados inferem a ação do precursor da serotonina sobre a resposta imune, desenvolvimento intestinal, digestão e absorção de nutrientes de frangos de corte.
Agradecimentos.- Ao Conselho Nacional de Desenvolvimento Científico e Tecnológico (CNPq, processo 472571/2011-7) pelo financiamento do projeto e a Fundação de Amparo a Pesquisa do Estado de São Paulo (FAPESP - processo 2011/15338-1) pela concessão da bolsa de estudo.

\section{REFERÊNCIAS}

Alvarez L.C., Barrera E.M. \& Gonzáles E.A. 1994. Evaluacion de promotores Del crescimiento para pollos de engorda. Vet. Méx. 24:141-44.

Alverdy J.C., Aoys E. \& Moss G.S. 1990. Effect of commercially available chemically defined liquid diets on the intestinal microflora and bacterial translocation from the gut. J. Parenter. Enteral Nutr. 14:1-6.

Andreatti Filho R.L., Silva E.N. \& Curi P.R. 1997. Ácidos orgânicos e microbiota cecal anaeróbia no controle da infecção experimental de frangos por Salmonella Typhimurium e Salmonella Enteritidis. Arq. Bras. Med. Vet. Zootec. 49:661-72.

Behmer A.O., Tolosa E.M.C. \& Freitas Neto A.G. 2003. Manual de Técnicas para Histologia Normal e Patológica. Editora Manole, São Paulo. 331p.

Cedraz-Mercez P.L., Almeida A.C., Thomaz C.M., Costa e Sousa R.H., Olivares E.L., Cortes W.S., Medeiros M.A. \& Reis L.C. 2007. Effect of L-5 Hydroxytryptophan on drinking behavior in Coturnix japonica (Temminck et Schlegel, 1849) (Galliformes:Aves): Involvement of rennin-angiotensin system. Braz. J. Biol. 67:771-776.

Coates M.D., Mahoney C.R., Linden D.R., Sampson J.E., Chen J., Blaszyk H., Crowell M.D., Sharkey K.A., Gershon M.D., Mawe G.M. \& Moses P.L. 2004. Molecular defects in mucosal serotonin content and decreased serotonin reuptake transporter in ulcerative colitis and irritable bowel syndrome. Gastroenterology 126:1657-64.

Corneli J. 2004. Avaliação de promotores de crescimento alternativos em substituição aos convencionais sobre o desempenho, características de carcaça e morfometria intestinal em frangos de corte. Dissertação de Mestrado em Zootecnia, Universidade Federal de Santa Maria, Santa Maria, RS. 37p.

Cunningham J.G. 2004. Tratado de Fisiologia Veterinária. Guanabara Koogan, Rio de Janeiro. 450p.

Deitch E.A., Xu D., Naruhn M.B., Deitch D.C., Lu Q. \& Marino A.A. 1995. Elemental diet and IV-TPN-induced bacterial transloaction is associated with loss of intestinal mucosal barrier function against bactéria. Annals of Surgery 221:299-307.

Dobrogsz W.J., Black B.L. \& Casas J.A. 1991. Delivery of viable Lactobacillus reuteri to the gastrintestinal tract of poultry. Poult. Sci. 70:158.

Ferreira A.J.P., Ferreira C.A., Petrella Neto R., Oliveira D.R. \& Nuremberger Junior R. \& Lopes V.C.B.A. 1998. Probiotics: benefits and efficiency in poultry production. Arqs Inst. Biológico, São Paulo, 65:139-49.

Fukumoto S., Tatewaki M. \& Yamada T. 2003. Short-chain fatty acids stimulate colonic transit via intraluminal 5HT release in rats. Am. J. Physiol. Regul. Integr. Comp. Physiol. 284:1269-1276.

Furlan R.L., Macari M. \& Luquetti B.C. 2004. Como avaliar os efeitos do uso de prebióticos, probióticos e flora de exclusão competitiva. Simpósio Técnico de Incubação, Matrizes de Corte e Nutrição, Balneário Camboriú, SC, p.6-28. (Resumo)

Hasler W.L. 2009. Serotonin and the GI tract. Curr. Gastroenterol. Rep. 11:383-391.

Khan W.I. \& Ghia J.E. 2010. Gut hormones: emerging role in immune activation and inflammation. Clin. Exp Immunol. 161:19-27.

Linden D.R., Chen J.X., Gershon M.D., Sharkey K.A. \& Mawe G.M. 2003. Serotonin availability is increased in mucosa of guinea pigs with TNBSinduced colitis. Am. J. Physiol. Gastrointest. Liver Physiol. 285: 207216.

Macari M. \& Maiorka A. 2000. Função gastrintestinal e seu impacto no rendimento avícola. Conferência Apinco de Ciência e Tecnologia Avícolas, Campinas, SP, p.161-74. (Resumo)

Maiorka A. 2001. Adaptações digestivas pós-eclosão. Conferência Apinco de Ciência e Tecnologia Avícola, Campinas, SP, p.141-52. (Resumo)

Manocha M. \& Khan W.I. 2012. Serotonin and GI disorders: an update on clinical and experimental studies. Clin. Transl. Gastroenterol. 3:1-6. 
Marchini C.F.P., Silva P.L., Nascimento M.R.B.M., Beletti M.E., Guimarães E.C. \& Soares H.L. 2009. Morfometria da mucosa duodenal em frangos de corte submetidos à temperatura ambiente cíclica elevada. Arq. Bras. Med. Vet. Zootec. 61:491-497.

Masseno A.P.B. 2012. Avaliação da fibrose endometrial e dos miofibroblastos nas endometoses ativa e inativa das éguas. Tese de Doutorado em Patologia Animal, Faculdade de Medicina Veterinária e Zootecnia, Universidade Estadual Paulista, Botucatu, SP. 100p.

Murarolli V.D.A. 2008. Efeito de probiótico, prebiótico e simbiótico sobre o desempenho, morfologia intestinal e imunidade de frangos de corte. Dissertação de Mestrado em Medicina Veterinária, Centro de Ciências Agrárias da Universidade de São Paulo, Pirassununga, SP. 101p.

Nakamura K., Sato T., Ohashi A., Tsurui H. \& Hasegawa H. 2008. Role of a serotonin precursor in development of gut microvilli. Am. J. Pathol. 172:333-344.

O`Hara J.R., Skinn A.C., Macnaughton W.K., Sherman P.M. \& Sharkey K.A. 2006. Consequences of Citrobacter rodentium infection on enteroendocrine cells and the enteric nervous system in the mouse colon. Cell Microbiol. 8:646-660.

0`Hara J.R., Ho W., Linden R.D., Mawe G.M. \& Sharkey K.A. 2004. Enteroendocrine cells and $5 \mathrm{HT}$ availability are altered in mucosa of guinea pigs with TNBS ileitis. Am. J. Physiol. Gastrointest. Liver Physiol. 287:998-1007.
Pelicano E.R.L., Souza P.A., Souza H.B.A., Oba A., Norkus E.A., Kodawara L.M. \& Lima T.M.A. 2003. Morfometria e ultra-estrutura da mucosa intestinal de frangos de corte alimentados com dietas contendo diferentes probióticos. Revta Port. Ciênc. Vet. 98:81-84.

Ramos L.S.N., Lopes J.B., Silva S.M.M.S., Silva F.E.S. \& Ribeiro M.N. 2011. Desempenho e histomorfometria intestinal de frangos de corte de $1 \mathrm{a}$ 21 dias de idade recebendo melhoradores de crescimento. Revta Bras. Zootec. 40:1738-1744.

Rocha T.S., Baptista A.A.S., Donato T.C., Milbradt E.L., Okamoto A.S., Rodrigues J.C.Z., Coppola M.P. \& Andreatti Filho R.L. 2012. Evaluation of in vitro and in vivo adhesion and immunomodulatory effect of Lactobacillus species strains isolated from chickens. Poult. Sci. 91:362-369.

SAS Institute 2009. SAS/STAT User`s Guide. Version 9.2. SAS Institute Inc, Cary, NC.

Schultz M., Munro K., Tannock G.W., Melchner I., Gotil C., Schwietz H., Scholmerich J. \& Rath H.C. 2004. Effects of feeding a probiotic preparation (SIM) containing insulin on the severity of colitis and on the composition of the intestinal microflora in HLA-B27 transgenic rats. Clin. Diagn. Lab. Immunol. 11:581-587.

Schwarz K.K., Furuya W.M., Natali M.R.M., Gaudezi M.C. \& Lima P.A.G. 2011. Mananoligossacarídeo em dietas para larvas de tilápia. Revta Bras. Zootec. 40:2634-2640. 